\title{
Augmentation bruit of the vertebral artery
}

\author{
C. MILLER FISHER \\ From the Department of Neurology, Massachusetts General Hospital, Boston, Mass., U.S.A.
}

Little has been written about bruits of the vertebral artery, and in general they are not made use of in clinical neurology. Even the characteristics of the bruit of vertebral stenosis remain inadequately depicted, for although it is at times inferred that bruits located in the supraclavicular region are due to stenosis of the lower vertebral artery and not to disease of the larger arteries nearby, proof of the site of origin has usually not been obtained. The vertebral bruit described in the present brief paper is of a different type, namely, that which is associated with an increased blood flow along one or both vertebral arteries.

When flow in a vessel is augmented beyond a certain limit, turbulence arises in the stream and the resultant vibration transmitted to the wall is heard with the stethoscope (Allen and Mustain, 1962). For vascular murmurs produced in this way, we suggest the term 'augmentation bruit' to connote the underlying increase in velocity and volume of blood flow. In the carotid system, bruits of this nature are well recognized and may be heard over the carotid arteries in the neck as well as over the eyeballs (Fisher, 1957) and skull.

Although augmentation bruits of the vertebral artery are not common, information about them should assist in the bedside interpretation of cerebrovascular disease, let alone furnish the answer to some heretofore puzzling bruits heard in the neck. Also clues to the haemodynamics of the cerebral blood flow are provided.

Augmentation bruits of the vertebral artery have been found in three conditions: (1) bilateral carotid artery occlusion with abundant basilar anastomotic flow to the middle and anterior cerebral territories; (2) subclavian steal; and (3) haemangioma of the brain-stem. Five representative cases will be described.

Typically, the bruit which is moderately loud is heard along the vertebral artery from the supraclavicular fossa to the mastoid region. The line of greatest intensity lies posterior to the carotid vessels, along the posterior border of the sternocleidomastoid muscle (Fig. 1). The bruit is often audible in the carotid region as well and then is usually interpreted as carotid in origin. At times carotid and vertebral bruits are both present and it may be difficult to distinguish the two by moving the stethoscope and judging the changing intensity. The sound is long and principally systolic but probably extends into diastole. It is not a continuous murmur. A thrill has not been felt. The patient is usually unaware of the noise.

Before concluding that the bruit arises in the vertebral artery, it is necessary to test the effect of digital compression of the common carotid artery. This should abolish sounds arising in the carotid artery while those in the vertebral system will be either unchanged or louder. If the result is still equivocal, both common carotid arteries may be briefly compressed at the same time during auscultation. We are not referring here in any way to vertebral bruits that are not present to start with and appear only upon carotid compression.

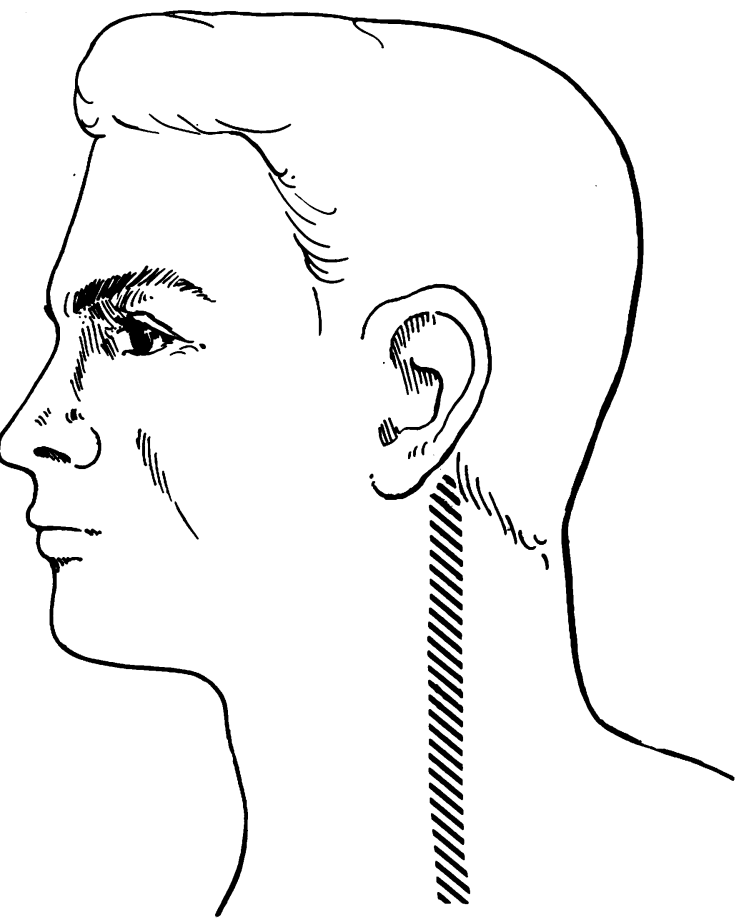

FIG. 1. Location of bruit. 


\section{CASE MATERIAL}

CASE 1 Case 1 was that of a man aged 69 with bilateral internal carotid artery occlusion. Six months and five months before admission, he suddenly lost consciousnesss without warning, the first time for three to five minutes, the second for about 20 seconds. There was no paralysis, numbness, dysarthria, or headache. Recovery was prompt. Shortly thereafter it was noted that the right pupil was larger than the left (right $7 \mathrm{~mm}$., left $3 \mathrm{~mm}$.) and the right pupil reacted sluggishly to light. On admission to hospital the patient had no complaints.

Neurological examination was within normal limits except for the right mydriasis and a questionable bilateral nasal hemianopia. The blood pressure was $160 / 80 \mathrm{~mm}$. Hg in the right arm and $140 / 60 \mathrm{~mm}$. $\mathrm{Hg}$ in the left. The systolic central retinal artery pressure was less than 10 units in each eye.

There were extremely loud systolic 'Sh-Sh' bruits over each common carotid artery bifurcation. A systolic bruit was heard more posteriorly on the left side along the posterior border of the sternocleidomastoid muscle. A moderately loud 'Ss-Ss' bruit was heard over the left orbit. Bruits were not present over the cheeks or temples. The carotid bruits ceased on compression of the common carotid arteries, but the bruit over the vertebral artery persisted and the ocular bruit became accentuated. The right carotid sinus was sensitive to gentle massage.

Carotid arteriograms showed complete occlusion of both internal carotid arteries with extensive filling of the middle cerebral and anterior cerebral territories via the ophthalmic arteries which were about $2 \mathrm{~mm}$. in diameter. The carotid siphons were filled for $1 \mathrm{~cm}$. proximal to the ophthalmic artery. Each external carotid artery was extremely stenotic at its origin accounting for the carotid systolic bruits.

A left vertebral arteriogram via brachial catheterization showed a huge $8 \mathrm{~mm}$. vertebral artery with good filling, of not only the basilar system but both middle cerebral and both anterior cerebral arteries as well. One posterior communicating artery was large, the other small. Presumably the left was larger, accounting for the ocular bruit on that side, but this could not be determined with certainty. The vertebral artery was not stenotic at its origin.

In this case there was good evidence that enhanced flow in the left vertebral artery was responsible for the cervical bruit. Enhanced flow via the left posterior communicating artery probably accounted for the ocular bruit, an indication that an ocular bruit does not always mean a patent carotid system.

CASE 2 Case 2 was that of a man, aged 50, who six months before admission while recuperating from a myocardial infarction, suddenly developed a right hemiplegia and aphasia. Improvement occurred in the next week but then he awakened with a recurrence of the weakness, particularly of the leg. He improved steadily thereafter and after six months had a mild dysphasia and a slight right hemiparesis.

The central retinal artery pressures were $5 / 20$ on the right, $42 / 92$ on the left. The blood pressure was $168 / 90$ $\mathrm{mm}$. Hg. The right pupil was $6 \mathrm{~mm}$., the left $4.5 \mathrm{~mm}$. Both reacted sluggishly to light. The superficial temporal vessels on both sides of the head were remarkably prominent, more so on the left. This had appeared after his stroke.

There was a moderately loud long 'fff-fff' bruit on the left side of the neck from the clavicular region to the level of the mastoid. It was loudest just below and posterior to the mastoid process. The bruit was at first interpreted incorrectly as arising in the carotid system. However, on compression of the left common carotid artery the bruit persisted. A faint sound was heard over the right carotid sinus, none on the left. There were no bruits over the eyes or skull.

Carotid angiograms showed complete occlusion of both internal carotid arteries in the region of the carotid sinus. Collateral filling of the carotid system via the external carotid and ophthalmic systems was minimal. A left vertebral angiogram via brachial catheterization demonstrated a large left vertebral artery up to $11 \mathrm{~mm}$. wide. The lower basilar artery was moderately stenosed. The posterior cerebral arteries filled well and in later films an unusually rich collateral flow was shown passing through the subarachnoid interarterial anastomoses from the posterior cerebral vessels to fill the middle and anterior cerebral systems on each side. Also a small amount of dye passed into one internal carotid artery through a posterioro communicating artery. There was no stenosis of the sube clavian artery or of the origin of the left vertebral artery?

CASE 3 Case 3 was that of a man aged 34 who suffere a subarachnoid haemorrhage in association with bilaterat hypoplastic internal carotid arteries. For six months the patient, a truck driver, had had episodes of sweating and a feeling of faintness on getting out of his truck after long journeys. One night while undressing for bed he suddenly lost consciousness and on admission to hospital was found to have bloody cerebrospinal fluid. Recovery was uneventful except for the development of a sixth nerve palsy. For one day after the haemorrhage he had been deaf bilaterally. Turning of the neck had never elicited any untoward effects.

On examination seven weeks later the only abnormalities were weak carotid pulsations bilaterally, and a moderately loud systolic bruit heard along the posterolateral aspect of the neck on the left side from just above the clavicle to the mastoid process. This bruit was not influenced by compression of the common carotid arteries, separately or together. There was no bruit over the skull or eyes, along the carotid arteries, or along the right vertebral artery. The superficial temporal pulses were normal. The central retinal artery diastolic pressure was 12 on the right, 25 on the left.

A right carotid angiogram showed a small common carotid artery and an external carotid artery of normal size. The internal carotid artery was unusually small, the region of the sinus measuring about $2 \mathrm{~mm}$. in diameter. The vessel was also small within the petrous bone and in the region of the cavernous sinus. Just distal to the origin of the ophthalmic artery a congerie of small vessels was seen lying along the sella turcica and extending laterally for a few centimetres. In a later phase both anterior cere- 
bral arteries filled faintly and possibly a twig of the right middle cerebral artery was visible. A left carotid arteriogram showed a similar picture except that the internal carotid was smaller than the right and could not be traced higher than the petrous bone.

A right vertebral angiogram showed a large vertebral artery and good filling of the basilar system. In addition the posterior communicating arteries filled extremely well and on later films both middle cerebral systems were visualized. What appeared to be a $1 \mathrm{~cm}$. saccular aneurysm lay at the bifurcation of the basilar artery. A vascular disorder almost identical with the findings in this case has been described previously (Fisher, 1959).

CASE 4 Case 4 was that of a man aged 72 who had been well except for two episodes of transient blindness of the left eye. The first attack had occurred one and a half years before and lasted approximately 36 hours. The second occurred three months before and lasted 24 to 48 hours.

On examination the blood pressure in the right arm was $120 / 80 \mathrm{~mm}$. $\mathrm{Hg}$, in the left $160 / 80 \mathrm{~mm}$. Hg. Loud bruits were heard over each carotid sinus in the neck, over each eyeball, and in the right supraclavicular fossa. In addition there was a loud murmur along the left vertebral artery from the clavicle to the mastoid process. During compression of each common carotid artery separately, this murmur persisted and was greatly accentuated whereas the corresponding ipsilateral carotid and ocular bruits ceased. The right radial pulse was delayed.

An arch aortogram via the left brachial artery showed occlusion of the proximal right subclavian artery and a remarkably abundant 'subclavian steal', dye that entered the left vertebral artery being diverted directly to the right subclavian artery via the right vertebral artery. The right internal carotid artery was moderately stenosed at its origin. The bruit in this case was clearly attributable to the subclavian steal.

CASE 5 Case 5 was that of a boy aged 13 who awakened one morning with a severe occipital headache. The cerebrospinal fluid was bloody. The headache recurred nine days later and was associated with vomiting. He complained of diplopia. Examination showed neck stiffness, horizontal nystagmus, and cerebellar ataxia of the left arm and leg. On auscultation there was a loud, low, rumbling murmur over each mastoid process and down along the region of the vertebral arteries. There was no bruit over the carotid sinuses or over the eyes. Arteriograms showed a large haemangioma within the posterior fossa involving chiefly the region of the right cerebellar hemisphere and the vermis.

This murmur was interpreted as due to increased blood flow in the vertebral arteries rather than to quickened venous flow.

\section{COMMENT}

Little need be added here. It is of interest that the bruit was heard in four cases on the left side, in one bilaterally, and in none on the right side alone.

The possibility that the bruits were determined by local factors and not primarily by increased blood flow was considered but the arteriograms ruled out atherosclerotic stenosis and bony osteoarthritic spurs. A rotated position of the neck was not a factor; and we have consistently failed to produce bruits in a series of patients by this manoeuvre.

\section{SUMMARY}

Five cases have been reported of a bruit arising in the vertebral artery as a result of enhanced blood flow. The term 'augmentation bruit' is suggested for this type of bruit.

\section{REFERENCES}

Allen N., and Mustian, V. (1962). Origin and significance of vascular murmurs of the head and neck. Medicine (Baltimore), 41, 227-247.

Fisher, C. M. (1957). Cranial bruit associated with occlusion of the internal carotid artery. Neurology (Minneap.), 7, 299-306.

- (1959). Early-life carotid artery occlusion associated with late intracranial hemorrage. Lab. Invest., 8, 680-700. 